\title{
Paridad y sintomatología ansiosa en beneficiarias de programas sociales de Lima Norte
}

\section{Claudia Cahui-Ramirez ${ }^{1}$}

RESUMEN: El presente estudio buscó determinar el grado de relación entre paridad y sintomatología ansiosa en mujeres beneficiarias de programas sociales de Puente Piedra y Los Olivos. El diseño fue transversal y de alcance explicativo. Se aplicaron los siguientes instrumentos: Nueva Escala de Ansiedad (NEA-72) y una ficha de datos sociodemográficos. La muestra estuvo compuesta por 230 mujeres de las cuales el 77,4\% presentó ansiedad clínicamente no significativa (ACNS) y el 22,6\% ansiedad clínicamente significativa (ACS). Tener de tres a cuatro hijos aumentó 2.16 veces más la posibilidad de padecer ACS, a diferencia de tener uno a dos hijos (OR:3.16;IC95\%:1.52-6.54). Asimismo, haber alcanzado una educación superior $(92.3 \%)$ se relaciona con ACNS. Por lo tanto, existe relación directa entre la paridad y sintomatología ansiosa, situación que puede agravarse ante un bajo nivel de escolaridad. No obstante, un alto nivel de instrucción podría ser un factor protector para la ACS.

PALABRAS CLAVE: Sintomatología ansiosa; Paridad; Mujeres; Programas sociales.

Citar como: Cahui C. Paridad y sintomatología ansiosa en beneficiarias de programas sociales de Lima Norte. CASUS. 2017;2(2):112-118. 


\section{INTRODUCCIÓN}

La ansiedad es un estado afectivo-emocional que se manifiesta de manera constante en las situaciones de la vida, como pueden ser la presentación de un trabajo importante o la proximidad de una fecha especial, siendo útil para estar alerta y organizar determinadas actividades. La ansiedad en estas circunstancias es transitoria (1). En cambio, para otras personas esta ansiedad no desaparece y sobrepasa su capacidad de adaptación manifestando malestar, síntomas psicosomáticos y convirtiéndose en un factor potencial de riesgo para el deterioro de la salud mental (2).

La ansiedad es el segundo trastorno mental más frecuente en América Latina y el Caribe (3). En el Perú los trastornos de ansiedad resultan más frecuentes en la población femenina (4). De la misma manera se registra en Lima Metropolitana y el Callao (5). Las investigaciones sobre la etiología de este fenómeno explican que se da por la interacción de un conjunto de factores que representan un riesgo para desarrollar este trastorno mental (6). Comprobándose que vivir en un contexto de desigualdad social, pobreza, baja calidad educativa y falta de un plan de vida se asocia a una maternidad temprana, lo cual podría propiciar la inclusión de estas madres a programas sociales (7). En consecuencia, se incrementaría el riesgo de padecer síntomas ansiosos al tener más hijos a los cuales atender y criar. En este sentido, se ha demostrado que padecer tres o más eventos estresantes aumentan la probabilidad en la mujer de experimentar ansiedad y/o comorbilidades (8). Por ello, desde la psicología es fundamental el aporte científico para generar mayor conocimiento sobre los factores de riesgo de la sintomatología ansiosa que pueden afectar la salud mental de las gestantes o madres.

En el Perú el promedio de hijos es 2.5 (9). Así mismo, la crianza o interacción con los hijos es considerado por un $30.3 \%$ de mujeres como el factor más estresante dentro de las actividades cotidianas (5). Es decir, la mujer se dedica a desarrollar múltiples actividades en el hogar, el trabajo y la comunidad, trabajando a la semana 23 horas con 35 minutos más que los padres en actividades domésticas. Esta responsabilidad se incrementa conforme existen más integrantes en la familia (10). A nivel internacional, se han realizado estudios relacionados a la paridad y ansiedad: las mujeres en la etapa postnatal presentan niveles significativos de ansiedad y comorbilidades. Este riesgo se incrementa si experimentan tres o más eventos estresantes (8). El desarrollo de actitudes y creencias irracionales sobre la crianza y los cuidados de un hijo pueden generar la aparición de un trastorno de ansiedad y comorbilidades (11). Se halló mayor sintomatología ansiosa, somática e insomnio en mujeres multíparas en comparación con las nulíparas (12), e incluso las mujeres en edad reproductiva son más vulnerables y la probabilidad de padecer un trastorno de ansiedad se incrementa de 2 a 3 veces más respecto a los hombres (13). En el contexto nacional, la realidad es similar, encontrándose que las mujeres pueden llegar a considerar a sus hijos como el factor más estresante (5) igualmente estados de ansiedad en madres beneficiarias de programas sociales (14).

Por otra parte, los programas sociales en el Perú fueron creados por un grupo de mujeres con la finalidad de proveer y alimentar a sus familias (15). Esta situación muestra la preocupación de estas madres con bajos recursos económicos, que puede incrementarse ante la existencia de más hijos que mantener. Actualmente los hallazgos científicos revelan que las madres experimentan acontecimientos vitales estresantes relacionados a los hijos (8) y que ser mujer es un factor de riesgo relacionado al trastorno de ansiedad (1).

En el Perú se ha implementado un programa de atención integral en el sector público de salud para la evaluación y detección temprana de problemas de salud mental en mujeres sexualmente activas o gestantes a cargo de un equipo multidisciplinario liderado por un(a) obstetra, que inicia el proceso y según su criterio profesional refiere a la paciente al departamento de psicología (16). Por lo tanto, es pertinente cuestionar qué tan acertado sea que las funciones de un psicólogo frente a los problemas de salud mental dependan del visto bueno de otro profesional de la salud, más aún en poblaciones vulnerables que requieren una atención psicológica especializada y constante.

Dada la importancia del contexto resulta relevante realizar estudios en la población femenina por estar más expuestas a sufrir este tipo de sintomatología y por la necesidad de identificar más factores de riesgo, con la finalidad de realizar una mejor intervención preventiva en salud mental. Teniendo en cuenta lo anterior, se determinó el grado de relación entre paridad y sintomatología ansiosa en mujeres beneficiarias de programas sociales de Puente Piedra y Los Olivos.

\section{MATERIALES Y MÉTODOS}

El diseño del estudio fue transversal y de alcance explicativo, realizándose un censo. La muestra estuvo 
conformada por 230 madres beneficiarias de tres programas sociales de Puente Piedra y Los Olivos: 137 mujeres corresponden al programa vaso de leche y 93 a un comedor popular. Se incluyeron a mujeres inscritas en la lista de empadronamiento de dichos programas y que tenían hijos nacidos vivos. Se excluyeron a mujeres menores de 18 años.

Para medir la variable dependiente, sintomatología ansiosa, se usó el instrumento Nueva Escala de Ansiedad (NEA)-72. La misma fue validada a nivel nacional y constituida por 72 ítems de tipo dicotómico (sí/no). El puntaje $\geq 39$ se atribuye a indicador de sintomatología ansiosa en mujeres y se categoriza en ansiedad clínicamente significativa (ACS) y no significativa (ACNS).

La variable paridad está definida por el número de hijos vivos hasta la actualidad (17), medida a través de la siguiente pregunta: ¿Cuántos hijos nacidos vivos ha tenido durante toda su vida? (18). La misma se categorizó en uno a dos hijos, de tres a cuatro y cinco o más. La edad fue clasificada en cuatro grupos etarios de 18 a 24 años, 25 a 44 años, 45 a 64 años y 65 años o más; estado civil, variable categórica (casada, conviviente, separada, divorciada, viuda y soltera); grado de instrucción, variable categórica (superior universitario, superior no universitario, secundaria y primaria) y lugar de nacimiento, variable categórica (sierra, costa, selva y Lima metropolitana). Para el análisis estadístico se utilizó el programa SPSS versión 20, calculando frecuencias y porcentajes. En el análisis bivariado se aplicó la prueba de Chi-cuadrado. Para la fuerza de asociación se calculó Odss Ratio (OR) crudo y ajustado mediante la regresión logística e intervalos de confianza (IC) al $95 \%$ con una significancia menor o igual a 0.05 . Del mismo modo se empleó la prueba de ajuste de Hosmer-Lemeshow.

Para recolectar los datos el estudio contó con la aprobación de un Comité de Ética Institucional. Asimismo, se respetó la confidencialidad mediante el anonimato de las encuestadas.

\section{RESULTADOS}

De las 230 mujeres beneficiarias de los programas sociales se encontró que el $45.2 \%$ tenía de 25 a 44 años de edad, el $34.3 \%$ convivía con su pareja, el $47.4 \%$ tuvo estudios secundarios y el $61.3 \%$ nació en Lima metropolitana. Respecto a la ansiedad un 77.4\% presentó una ACNS y el $22.6 \%$ ACS. Asimismo el $68.3 \%$ de las mujeres tenía de uno a dos hijos (ver tabla 1).

\section{Tabla 1. Descripción de las variables en mujeres}

\begin{tabular}{|c|c|c|}
\hline & f & (\%) \\
\hline \multicolumn{3}{|c|}{ Sintomatología ansiosa } \\
\hline No & 178 & 77.4 \\
\hline Sí & 52 & 22.6 \\
\hline \multicolumn{3}{|l|}{ Paridad } \\
\hline $1-2$ & 157 & 68.3 \\
\hline $3-4$ & 59 & 25.6 \\
\hline 5 o más & 14 & 6.1 \\
\hline \multicolumn{3}{|l|}{ Edad } \\
\hline $18-24$ & 31 & 13.5 \\
\hline $25-44$ & 104 & 45.2 \\
\hline $45-64$ & 89 & 38.7 \\
\hline 65- a más & 6 & 2.6 \\
\hline \multicolumn{3}{|c|}{ Grado de instrucción } \\
\hline Primaria & 25 & 10.9 \\
\hline Secundaria & 106 & 47.4 \\
\hline Técnico & 44 & 19.1 \\
\hline Superior & 52 & 22.6 \\
\hline \multicolumn{3}{|l|}{ Estado civil } \\
\hline Casada & 74 & 32.2 \\
\hline Conviviente & 79 & 34.3 \\
\hline Divorciada & 8 & 3.5 \\
\hline Separada & 35 & 15.2 \\
\hline Soltera & 27 & 11.7 \\
\hline Viuda & 7 & 3.0 \\
\hline
\end{tabular}


En la tabla 2 se muestra que tener cinco o más hijos (42.9\%) se asoció con la presencia de ansiedad, mientras que tener de uno a dos hijos (84.1\%) se asocia con ACNS. Asimismo tener educación primaria (56\%) se asoció con ACS y tener un grado superior $(92.3 \%)$ se asoció con ACNS. El resto de las variables no mostró significancia.

Tabla 2. Asociación entre paridad y variables sociodemográficas y sintomatología ansiosa

\begin{tabular}{|c|c|c|c|}
\hline & $\begin{array}{c}\text { No } \\
n(\%)\end{array}$ & $\begin{array}{c}\mathrm{Si} \\
\mathrm{n}(\%)\end{array}$ & $p$-value \\
\hline $\begin{array}{l}\text { Paridad } \\
1-2 \\
3-4 \\
5 \text { o más }\end{array}$ & $\begin{array}{c}132(84.1) \\
38(64.4) \\
8(54.1)\end{array}$ & $\begin{array}{c}25(15.9) \\
21(35.6) \\
6(42.9)\end{array}$ & 0.002 \\
\hline $\begin{array}{l}\text { Edad } \\
18-24 \\
25-44 \\
45-64 \\
65-\text { a más }\end{array}$ & $\begin{array}{c}26(83.9) \\
83(79.8) \\
66(74.2) \\
3(50.0)\end{array}$ & $\begin{array}{c}5(16.1) \\
21(20.2) \\
23(25.8) \\
3(50.0)\end{array}$ & 0.241 \\
\hline $\begin{array}{l}\text { Grado de in } \\
\text { Primaria } \\
\text { Secundaria } \\
\text { Técnico } \\
\text { Superior }\end{array}$ & $\begin{array}{l}\text { ión } \\
11(44.0) \\
82(75.2) \\
37(84.1) \\
48(92.3)\end{array}$ & $\begin{array}{c}14(56.0) \\
27(24.8) \\
7(15.9) \\
4(7.7)\end{array}$ & 0.000 \\
\hline $\begin{array}{l}\text { Estado civil } \\
\text { Casada } \\
\text { Conviviente } \\
\text { Divorciada } \\
\text { Separada } \\
\text { Soltera } \\
\text { Viuda }\end{array}$ & $\begin{array}{c}56(75.79 \\
59(74.7) \\
6(75.0) \\
32(91.4 \\
21(77.8) \\
4(57.1)\end{array}$ & $\begin{array}{c}18(24.3) \\
20(25.3) \\
2(25.0) \\
3(8.6) \\
6(22.2) \\
3(42.9)\end{array}$ & 0.300 \\
\hline
\end{tabular}

En la tabla 3 se muestra la fuerza de asociación entre paridad y sintomatología ansiosa tanto en el modelo no ajustado (crudo) como el ajustado (multivariado). Según el modelo ajustado el tener de tres a cuatro hijos aumenta 2.16 veces más la posibilidad de padecer ACS, a diferencia de tener uno a dos hijos
(OR: 3.16;IC95\%:1.52-6.54). Por otro lado, haber alcanzado un grado de instrucción superior resultó ser un factor protector frente a los síntomas ansiosos en comparación con tener instrucción primaria (OR: $0.05 ; \mathrm{IC} 95 \% ; 0.01-0.22)$. La bondad de ajuste del modelo fue de 0.644 .

Tabla 3. Análisis multivariado entre la paridad, grado de instrucción y sintomatología ansiosa

\begin{tabular}{lcccccc}
\hline & \multicolumn{3}{c}{ Modelo Crudo } & \multicolumn{4}{c}{ Modelo Ajustado } \\
& OR & IC $95 \%$ & p-value & OR & IC 95\% & p-value \\
\hline Paridad & & & & & & - \\
$1-2$ & 1.0 & - & - & 1.0 & - & - \\
$3-4$ & 2.92 & $1.47-5.78$ & 0.002 & 3.16 & $1.52-6.54$ & 0.002 \\
5 o más & 3.96 & $1.26-12.4$ & 0.018 & 1.11 & $0.27-4.60$ & 0.876 \\
& & & & & & \\
Grado de instrucción & 1.0 & - & - & 1.0 & - & - \\
Primaria & 0.26 & $0.10-0.64$ & 0.003 & 0.21 & $0.07-0.62$ & 0.005 \\
Secundaria & 0.15 & $0.05-0.46$ & 0.001 & 0.14 & $0.38-0.51$ & 0.003 \\
Técnico & 0.06 & $0.02-0.24$ & 0.000 & 0.05 & $0.01-0.22$ & 0.000 \\
Superior & & & & & &
\end{tabular}




\section{DISCUSIÓN}

Los datos del estudio muestran que un $22.6 \%$ de las mujeres presentó ansiedad clínicamente significativa y un $68.3 \%$ del total tenía de uno a dos hijos. Asimismo, un aumento en la cantidad de hijos se asocia positivamente con la ACS y poseer un grado de instrucción superior se muestra como un factor protector frente a la sintomatología ansiosa.

Un aumento en la cantidad de hijos se asocia positivamente con la ACS. Este resultado es similar a un estudio que evidencia que un mayor número de hijos se asocia a síntomas de ansiedad en comparación con las mujeres que tienen menos o no tienen descendencia (12). Cabe resaltar que las participantes residen en distritos de Lima Norte en los que se concentra una de las poblaciones con mayor pobreza. Por tal motivo pudiera existir una relación entre las beneficiarias y la presencia de ansiedad (14). Las mujeres que participan en los programas sociales probablemente experimentan acontecimientos vitales estresantes relacionados a no tener un trabajo estable, la crianza de los hijos y dificultades del sustento económico, etc. Incluso tener simultáneamente tres o más de estos eventos estresantes aumenta la probabilidad de presentar un trastorno de ansiedad o incrementar los síntomas ansiosos (8). Sumado a ello, la crianza o interacción con los hijos es considerado como el factor más estresante dentro de las actividades cotidianas (5). Esto podría deberse a que las mujeres que tienen más hijos son más perceptivas de los problemas que presentan integrantes de su familia y amigos generando mayor susceptibilidad frente al estrés en las personas de su entorno (19). Del mismo modo, las madres cambian sus actitudes y pueden desarrollar creencias irracionales relacionadas con la presencia de ansiedad (11).

Otro hallazgo importante del estudio fue que el grado de instrucción superior funciona como un factor protector frente a la ACS. Probablemente se deba a que las madres con un mayor grado de instrucción tengan una mayor estabilidad económica y conocimientos sobre la planificación familiar (20). Por estas razones presentan mayores posibilidades de cumplir con su número ideal de hijos, tener un bebé sano y asegurar un mejor rendimiento académico y desarrollo de habilidades cognitivas en sus hijos en comparación con madres de nivel educativo inferior (20). Por ende, puede que las mujeres profesionales o con educación superior tengan mayor acceso al conocimiento sobre su salud mental y busquen ayuda profesional con el fin de prevenir o tratar la sintomatología ansiosa y otros problemas relacionados a su salud.

Los resultados del estudio se deben interpretar considerando las siguientes limitaciones: no incluir variables sociodemográficas como la presencia del padre de familia, el tipo de trabajo de la madre, otros factores laborales, el número de integrantes en el hogar y antecedentes médicos u obstétricos. En cuanto al diseño se pueden presentar sesgo de información, de memoria y temporalidad. Se recomienda en futuros estudios profundizar la temática incluyendo otras variables como la satisfacción marital, los estilos de crianza e información relativa a los padres de familia para determinar su influencia sobre la sintomatología ansiosa.

\section{CONCLUSIONES}

El aumento en la cantidad de hijos está asociado directamente con la ACS. Mientras que tener un grado de instrucción superior es un factor protector frente a la sintomatología ansiosa. Además, presentar un mayor número de hijos y tener un bajo nivel de escolaridad aumentan la probabilidad de presentar ACS. Por ello, se sugiere orientar la capacidad de convocatoria que poseen las líderes de estos programas para que se organicen y soliciten a los centros de salud o autoridades municipales, la presencia de un grupo multidisciplinario que acuda a sus locales y focalice la intervención en salud mental según los niveles de atención que correspondan. Asimismo, que estas mujeres puedan recibir la información adecuada con respecto a su salud desde un enfoque que contemple de forma consciente el valor de la vida humana y el compromiso de cuidar a los hijos que engendran. 


\section{REFERENCIAS BIBLIOGRÁFICAS}

1. National Institute of Mental Health [internet]. Anxiety Disorders. United States: National Institute of Health; 2016 [citado feb 2016]. Disponible en:https://www.nimh.nih.gov/health/ topics/anxiety-disorders/index.shtml

2. Organización Panamericana de la Salud. Depresión y otros trastornos mentales comunes. Estimaciones sanitarias mundiales. Washington, D.C.:OPS; 2017.

3. Organización Panamericana de la Salud y Organización Mundial de la Salud. Día mundial de la salud mental: la depresión es el trastorno mental más frecuente. Buenos Aires: OMS; 2012.

4. Fiestas F, Piazza M. Prevalencia de vida y edad de inicio de trastornos mentales en el Perú urbano: Resultados del estudio mundial de salud mental, 2005. Rev Peru Med Exp Salud Pública. 2014;31(1):39-47.

5. Instituto Especializado de Salud Mental "Honorio Delgado- Hideyo Noguchi”. Anales de Salud Mental. Lima: Instituto Nacional de Salud Mental; 2002.

6. Organización Mundial de la Salud y Centro de Investigación de Prevención de las Universidades de Nijmegen y Maastricht. Prevención de los trastornos mentales: intervenciones efectivas y opciones de políticas. Ginebra: OMS; 2004.

7. Banco Mundial. Embarazo adolescente y oportunidades en América Latina y el Caribe: sobre maternidad temprana, pobreza y logros económicos. Washington, DC.: Banco Mundial; 2012.
8. Yelland J, Sutherland G, Brown S Postpartum anxiety, depression and social health: findings from a population-based survey of Australian women. BioMed Central. 2010; 10:771.

9. Instituto Nacional de Estadística e Informática. Encuesta Demográfica y de Salud Familiar 2014. Lima: Instituto Nacional de Estadística e Informática; 2015.

10. Carrillo P. La familia peruana: ¿Espacio de Igualdad? Lo que nos revela la Encuesta del Uso del Tiempo y los desafíos para las políticas públicas. 2012.

11. Sockol L, Epperson C, Barber J. The relationship between maternal attitudes and symptoms of depression and anxiety among pregnant and postpartum first-time mothers. Arco Womens Ment Salud. 2014;17(3):199-212.

12. Matud MP. Diferencias de género en los síntomas más comunes de salud mental en una muestra de residentes en Canarias, España. Rev Mex Psi. 2005;22(2):395-403.

13. Arenas C, Puigcerver A. Diferencias entre hombres y mujeres en los trastornos de ansiedad: una aproximación psicobiológica. Escritos de Psicología. 2009;3(1):20-29.

14. Delgado E. Ansiedad estado-rasgo en madres del programa Vaso de leche de la zona urbana del distrito de Chiclayo y de la zona rural del distrito de Lambayeque, 2013 [tesis de pregrado]. Chiclayo:Universidad Católica Santo Toribio de Mogrovejo. Facultad de Medicina-Escuela de Psicología; 2015.
15. Angulo N. Comedores populares: seguridad alimentaria y ejercicio de ciudadanía en el Perú. 2004.

16. Norma técnica de salud para la atención integral de salud materna. Resolución Ministerial, n827-2013, (24-12- 2013).

17 Kozuki N, Lee A, Silveira M, Sania A, Vogel J, Adair L, et al. The associations of parity and maternal age with small-for-gestational-age, preterm, and neonatal and infant mortality: a meta-analysis. BMC Public Health. 2013;13(3).

18. Instituto Nacional de Estadística e Informática. Encuesta demográfica y de salud familiar [ENDES-2010]. Cuestionario Individual. Lima: INEI; 2010.

19. Kessler R, McLeod J. Sex differences in vulnerability to undesirable life events. Ame Soc Rev. 1984; 49: 620-631.

20. Livingston G, Cohn D. Long-Term Trend Accelerates During Recession Record Share of New Mothers are College Educated. Washington, D.C.: Pew Research Center; 2013. Serie 202.419.4372. 


\section{Parity and anxious symptomatology in beneficiaries of social programs of North Lima}

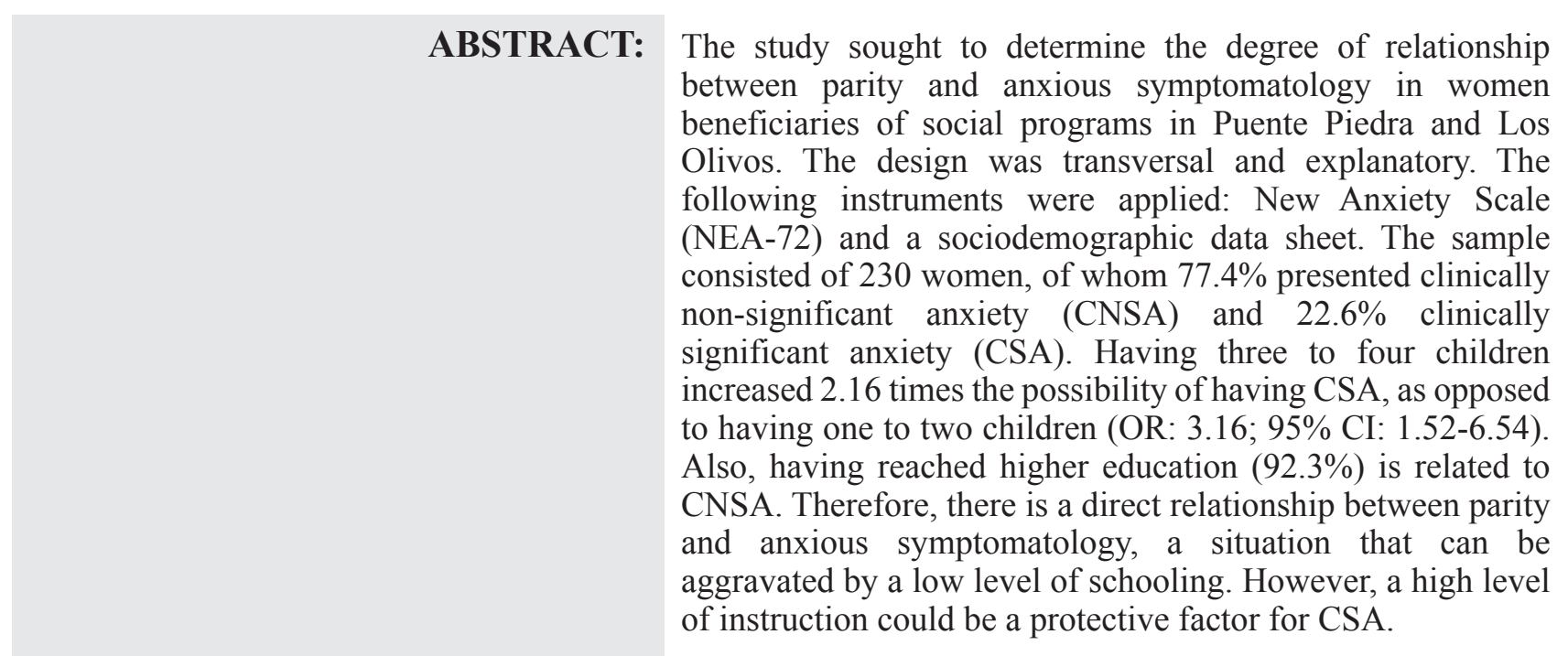

KEY WORDS: Anxious symptomatology; Parity; Women; Social programs. 